\title{
FIXED POINTS OF ENDOMORPHISMS OF CERTAIN FREE PRODUCTS
}

\author{
PEDRO V. Silva ${ }^{1}$
}

\begin{abstract}
The fixed point submonoid of an endomorphism of a free product of a free monoid and cyclic groups is proved to be rational using automata-theoretic techniques. Maslakova's result on the computability of the fixed point subgroup of a free group automorphism is generalized to endomorphisms of free products of a free monoid and a free group which are automorphisms of the maximal subgroup.
\end{abstract}

Mathematics Subject Classification. 20M05, 20F10.

\section{INTRODUCTION}

Gersten proved in the eighties that the fixed point subgroup of a free group automorphism $\varphi$ is finitely generated [10]. Using a different approach, Cooper gave an alternative proof, proving also that the fixed points of the continuous extension of $\varphi$ to the boundary of the free group is in some sense finitely generated [9]. Bestvina and Handel achieved in 1992 a major breakthrough through their innovative train track techniques, bounding the rank of the fixed point subgroup and the generating set for the infinite fixed points [3]. Their approach was pursued by Maslakova in 2003 to prove that the fixed point subgroup can be effectively computed [16].

Gersten's result was generalized to further classes of groups and endomorphisms in subsequent years. Goldstein and Turner extended it to monomorphisms of free groups [11], and later to arbitrary endomorphisms [12]. Collins and Turner extended it to automorphisms of free products of freely indecomposable groups [8], and recently Sykiotis to monomorphisms [21]. The interested reader can find more information in Ventura's excellent survey [22].

Keywords and phrases. Endomorphisms, fixed points, free products.

1 Centro de Matemática, Faculdade de Ciências, Universidade do Porto, R. Campo Alegre 687, 4169-007 Porto, Portugal. pvsilva@fc.up.pt 
Cassaigne and the author developed in [6] an approach to the study of monoids defined by special confluent rewriting systems (SC monoids) that preserves some of the features of the free group case and contains free products of cyclic groups as a particular case, as well as the partially reversible monoids introduced in [19]. In fact, the undirected Cayley graph of these monoids is hyperbolic and there exists a nice compact completion for the prefix metric. Uniformly continuous endomorphisms, algorithmically characterized in [6], admit a continuous extension to the boundary. In [7], the same authors used this approach to study the dynamics of infinite periodic points for two classes of endomorphisms of the monoids in question.

In [20], the author proved that the fixed point submonoids of a large class of uniformly continuous endomorphisms of SC monoids are rational (actually finitely generated in the group case), obtaining also results of the same flavour for infinite fixed points. In the group case, new results were obtained for infinite fixed points of monomorphisms of free products of cyclic groups (c-free groups).

In the present paper, we go beyond the uniform continuity restriction of [20], which is equivalent to injectivity in the group case. Section 3 is devoted to the problem of proving that the fixed point submonoid is rational, generalizing Goldstein and Turner's proof for free group endomorphisms. This involves facing some technical difficulties brought by the existence of finite order elements. We obtain thus a fully automata-theoretic proof of a result that follows also from previous results by Sykiotis [21] in the particular cases of monomorphisms or symetric endomorphisms. Another advantage of our proof is that it may offer some insight into the algorithmic aspects of the problem.

In Section 4, we discuss computability of the fixed point submonoid, the reference being of course Maslakova's result on free group automorphisms [16]. We generalize this result to endomorphisms of free products of a free monoid and a free group whose restriction to the group is an automorphism.

\section{Preliminaries}

Given a monoid $M$, we denote by Rat $M$ the set of all rational subsets of $M$, i.e., the smallest family of subsets of $M$ containing the finite sets and closed under union, product and the star operator ( $X^{*}$ denotes the submonoid of $M$ generated by $X \subseteq M)$. For details on rational languages, the reader is referred to $[2,18]$.

In the particular case of a free monoid $M=A^{*}$, a combinatorial description in terms of finite automata is usually preferred. We define a (finite) $A$-automaton to be a quadruple $\mathcal{A}=\left(Q, q_{0}, T, E\right)$ where $Q$ is a (finite) set, $q_{0} \in Q$ is the initial vertex, $T \subseteq Q$ are the terminal vertices and $E \subseteq Q \times A \times Q$. A nontrivial path in $\mathcal{A}$ is a sequence

$$
p_{0} \stackrel{a_{1}}{\longrightarrow} p_{1} \stackrel{a_{2}}{\longrightarrow} \ldots \stackrel{a_{n}}{\longrightarrow} p_{n}
$$

with $\left(p_{i-1}, a_{i}, p_{i}\right) \in E$ for $i=1, \ldots, n$. Its label is the word $a_{1} \ldots a_{n} \in A^{+}=$ $A^{*} \backslash\{1\}$. It is said to be a successful path if $p_{0}=q_{0}$ and $p_{n} \in T$. We consider 
also the trivial path $p \stackrel{1}{\longrightarrow} p$ for $p \in Q$. It is successful if $p=q_{0} \in T$. The language recognized by $\mathcal{A}$ is

$$
L(\mathcal{A})=\left\{w \in A^{*} \mid w \text { labels a successful path in } \mathcal{A}\right\} .
$$

The classical Kleene's Theorem states that $L \subseteq A^{*}$ is rational if and only if $L=$ $L(\mathcal{A})$ for some finite $A$-automaton $\mathcal{A}$. If we replace letters by rational languages as labels of edges (the resulting language being the union of the languages obtained by taking all the possible choices for each edge label), we remain within the realm of rational languages. Note that, if we fix a homomorphism $\pi: A^{*} \rightarrow M$, then Rat $M=\left(\operatorname{Rat} A^{*}\right) \pi$ and so the rational subsets of $M$ can be defined through finite automata.

Let $\mathcal{A}=\left(Q, q_{0}, T, E\right)$ be an $A$-automaton. We say that $\mathcal{A}$ is

- deterministic if $(p, a, q),(p, a, r) \in E$ implies $q=r$;

- complete if there exist edges with arbitrary label starting at every vertex;

- accessible if there exist paths from $q_{0}$ to any arbitrary vertex.

Another case that will be relevant for us is the case of $M$ being a group, when we have the following result of Anissimov and Seifert:

Proposition 2.1 ([18] Prop. II.6.2). Let $H$ be a subgroup of a group G. Then $H \in \operatorname{Rat} G$ if and only if $H$ is finitely generated.

A finite rewriting system is a formal expression $\langle A \mid R\rangle$, where $A$ is a finite alphabet and $R$ a finite subset of $A^{*} \times A^{*}$. The elements of $R$ are called rules. Once $R$ is fixed, for given $u, v \in A^{*}$, we write $u \longrightarrow v$ if

$$
u=x r y, \quad v=x s y
$$

for some $x, y \in A^{*}$ and $(r, s) \in R$. We denote by $\stackrel{*}{\longrightarrow}$ the reflexive and transitive closure of the relation $\longrightarrow$. The congruence on $A^{*}$ generated by $R$ will be denoted by $R^{\sharp}$. Note that $R^{\sharp}$ coincides with the relation $\stackrel{*}{\longrightarrow}$ defined with respect to $R \cup R^{-1}$. The quotient $M=A^{*} / R^{\sharp}$ is said to be the monoid defined by the rewriting system $R$. We denote by $\pi$ the canonical homomorphism $A^{*} \rightarrow M$.

A rewriting system $\langle A \mid R\rangle$ is said to be

- special if $R \subseteq A^{+} \times\{1\}$;

- confluent if, whenever $u \stackrel{*}{\longrightarrow} v$ and $u \stackrel{*}{\longrightarrow} w$, there exists $z \in A^{*}$ such that $v \stackrel{*}{\longrightarrow} z$ and $w \stackrel{*}{\longrightarrow} z$ :

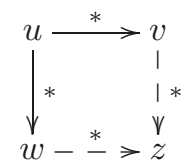

We shall refer to a monoid defined by a finite special confluent rewriting system as an $S C$ monoid. An important case is given by free groups. Indeed, the free group on $A$, denoted by $F G_{A}$, is defined by

$$
\left\langle A \cup A^{-1} \mid a a^{-1} \rightarrow 1, a^{-1} a \rightarrow 1(a \in A)\right\rangle,
$$

where $A^{-1}$ denotes a set of formal inverses of $A$. 
Let $\langle A \mid R\rangle$ be a special confluent rewriting system. We say that $w \in A^{*}$ is irreducible (with respect to $R$ ) if $w \notin \cup_{(r, s) \in R} A^{*} r A^{*}$. For every $u \in A^{*}$, there is exactly one irreducible $v \in A^{*}$ such that $u \stackrel{*}{\longrightarrow} v$ : existence follows from $R$ being lengthreducing, and uniqueness from confluence. We denote this unique irreducible word by $\bar{u}$. It is well known (see [5]) that the equivalence

$$
u \pi=v \pi \Leftrightarrow \bar{u}=\bar{v}
$$

holds for all $u, v \in A^{*}$, hence $\overline{A^{*}}=\left\{\bar{u} \mid u \in A^{*}\right\}$ constitutes a set of normal forms for the monoid $M=A^{*} / R^{\sharp}$.

A generalized version of the classical Benois' Theorem states that rational languages are preserved by reduction:

Theorem $2.2([1])$. Let $\langle A \mid R\rangle$ be a finite special confluent rewriting system and let $L \subseteq A^{*}$ be rational. Then $\bar{L}$ is rational and effectively constructible from $L$.

If $M$ and $M^{\prime}$ are defined respectively by the rewriting systems $\langle A \mid R\rangle$ and $\left\langle A^{\prime} \mid R^{\prime}\right\rangle$, then the free product $M * M^{\prime}$ is defined by $\left\langle A \cup A^{\prime} \mid R \cup R^{\prime}\right\rangle$, taking $A^{\prime}$ disjoint from $A$. We can now introduce the crucial concept of c-free group: a group is said to be $c$-free if it is a free product of cyclic groups. Since every free group is a free product of infinite cyclic groups, the class of c-free groups extends the class of free groups.

We denote the monoid of endomorphisms (respectively group of automorphisms) of $M$ by $\operatorname{End} M$ (respectively Aut $M$ ). Given $\varphi \in \operatorname{End} M$, let

$$
\operatorname{Fix} \varphi=\{u \in M \mid u \varphi=u\} \text {. }
$$

We say that Fix $\varphi$ is the submonoid of fixed points of $\varphi$. Note that Fix $\varphi$ is a group if $M$ is a group.

\section{RAtionality}

It is easy to determine which SC monoids can be embedded into some group. Following [17], we say that a monoid $M$ is directly finite if

$$
\forall x, y \in M \quad x y=1 \Rightarrow y x=1 .
$$

We recall that the bicyclic monoid is the SC monoid defined by the rewriting system $\langle a, b \mid a b \rightarrow 1\rangle$.

Proposition 3.1. Let $M$ be an SC monoid. Then the following conditions are equivalent:

(i) $\quad M$ is embeddable into some group;

(ii) $M$ contains no bicyclic submonoid;

(iii) $M$ is directly finite;

(iv) $M$ is a free product of a free monoid and cyclic groups. 
Proof. (i) $\Rightarrow$ (ii). Since a bicyclic monoid contains infinitely many idempotents $\left(b^{n} a^{n}\right.$ for the standard rewriting system).

(ii) $\Rightarrow$ (iii). By [15] Section VI.3.

(iii) $\Rightarrow$ (iv). Assume that $M$ is defined by the finite special confluent rewriting system $\langle A \mid R\rangle$. Let $A_{1}$ be the set of generators which occur in some relator of $R$, and write $A_{0}=A \backslash A_{1}$. Let $a \in A_{1}$. Then $R$ has some relator of the form uav $\rightarrow 1$ for some $u, v \in A^{*}$. Since $M$ is directly finite, it follows that $(v u a) \pi=1=(a v u) \pi$, hence $a \pi$ is invertible in $M$ and so $A_{1}^{*} \pi$ is a subgroup of $M$. Since $A_{1}^{*} \pi$ is defined by the finite special confluent rewriting system $\left\langle A_{1} \mid R\right\rangle$, we get a free product decomposition $M=A_{0}^{*} * A_{1}^{*} \pi$. By [20] Proposition 6.1, every SC group is c-free and so (iv) holds.

(iv) $\Rightarrow$ (i). We can extend the canonical embedding of $A^{*}$ in $F G_{A}$ to an embedding of $A^{*} * G$ into $F G_{A} * G$.

We adapt now Goldstein and Turner's proof [11] to c-free groups.

Theorem 3.2. Let $\varphi$ be an endomorphism of a finitely generated c-free group. Then Fix $\varphi$ is finitely generated.

Proof. Let $G$ be a finitely generated c-free group. Clearly, $G$ can be defined by a finite rewriting system of the form $\langle A \mid R\rangle$, where $A=A_{0} \cup A_{1} \cup A_{1}^{-1}$ and there exists $m_{a} \geq 2$ for every $a \in A_{0}$ such that

$$
R=\left\{\left(a^{m_{a}}, 1\right) \mid a \in A_{0}\right\} \cup\left\{\left(a a^{-1}, 1\right),\left(a^{-1} a, 1\right) \mid a \in A_{1}\right\} .
$$

Since the unique overlapping of relators we can get proceeds from the free group relators $a a^{-1}, a^{-1} a$, it follows easily that this special rewriting system is confluent, hence $G$ is an SC-monoid.

Let $\varphi$ be an endomorphism of $G$ and let $\pi: A^{*} \rightarrow G$ denote the canonical morphism. For every $a \in A_{0}$, write $a^{-1}=a^{m_{a}-1}$. Write also $\left(a^{-1}\right)^{-1}=a$ for every $a \in A_{1}$. Define $u^{-1}$ inductively for every $u \in A^{*}$ through

$$
1^{-1}=1, \quad(v a)^{-1}=a^{-1} v^{-1}\left(v \in A^{+}, a \in A\right) .
$$

Clearly, $\left(u^{-1}\right) \pi=(u \pi)^{-1}$ for every $u \in A^{*}$.

Clearly, $A^{*}$ acts on $G$ through $g a=g(a \pi)$. For every $g \in G$, let $Q(g)=\overline{g^{-1}(g \varphi)}$. Note that $g \in \operatorname{Fix} \varphi$ if and only if $Q(g)=1$. We define an $A$-automaton $\mathcal{A}_{\varphi}=$ $(Q, 1,1, E)$ by

$$
\begin{aligned}
& Q=\{Q(g) \mid g \in G\} ; \\
& E=\{(Q(g), a, Q(g a)) \mid g \in G, a \in A\} .
\end{aligned}
$$

Clearly, $\mathcal{A}_{\varphi}$ is a complete accessible deterministic automaton and

$$
L\left(\mathcal{A}_{\varphi}\right)=(\operatorname{Fix} \varphi) \pi^{-1} .
$$

We define a subautomaton $\mathcal{A}_{\varphi}^{\prime}=\left(Q, 1,1, E^{\prime}\right)$ by taking

$$
E^{\prime}=\{(p, a, q) \in E \mid a q \text { is irreducible }\} .
$$


Let $d_{\varphi}=\max \{|a \varphi| ; a \in A\}$ and $m_{R}=\max \left(\{2\} \cup\left\{m_{a} \mid a \in A_{0}\right\}\right)$. Note that, for all $u \in \overline{A^{*}}$ and $a \in A$, the suffix of $u$ involved in the reduction of $u(a \varphi)$ has length at most $\left(m_{R}-1\right) d_{\varphi}$ (since each letter of $a \varphi$ can erase at most $m_{R}-1$ letters of $u$ ).

We show that

$$
\forall p \in Q \quad|p|>\left(m_{R}-1\right) d_{\varphi} \Rightarrow p \text { has outdegree } \leq 1 \text { in } \mathcal{A}_{\varphi}^{\prime} .
$$

Let $p \in Q$ be such that $|p|>\left(m_{R}-1\right) d_{\varphi}$. Suppose that $(p, a, q),\left(p, b, q^{\prime}\right) \in E^{\prime}$ are distinct edges. Since $\mathcal{A}_{\varphi}$ is deterministic, we have $a \neq b$. It suffices to show that $p \in a A^{*}$. By symmetry, also $p \in b A^{*}$ and we reach the required contradiction.

Suppose that $p=c u$ with $c \in A \backslash\{a\}$. Then $q=\overline{a^{-1} c u(a \varphi)}$. Since $c \neq a$, then $a^{-1} c u$ is irreducible. Now $|u| \geq\left(m_{R}-1\right) d_{\varphi}$ implies that $a^{-1} c$ remains untouched in the reduction of $a^{-1} c u(a \varphi)$. Hence $q=a^{-1} \overline{c u(a \varphi)}$ and so $a q$ is reducible, contradicting $(p, a, q) \in E^{\prime}$. Thus $p \in a A^{*}$ and so (3.1) holds.

Given $q \in Q$, let the depth of $q$, denoted by $\operatorname{dep}(q)$, be the length of the shortest path $1 \longrightarrow q$ in $\mathcal{A}_{\varphi}$. Since $\mathcal{A}_{\varphi}$ is accessible, $\operatorname{dep}(q)$ is well defined.

Fix $s_{0}>1+\left(m_{R}-1\right) d_{\varphi}$ such that $s_{0} \geq m_{a}+\left(m_{R}-1\right)\left|a^{i} \varphi\right|$ for all $a \in A_{0}$ and $i \in\left\{1, \ldots, m_{a}-1\right\}$, and let

$$
s=m_{R}+\max \left\{\operatorname{dep}(p) \mid p \in Q \text { and }|p|<s_{0}\right\} .
$$

It follows that

$$
\operatorname{dep}(p)>s-m_{R} \Rightarrow|p| \geq s_{0}>1+\left(m_{R}-1\right) d_{\varphi}
$$

holds for every $p \in Q$.

Our proof of Theorem 3.2 requires the following intermediate result:

Lemma 3.3. If $(p, a, q) \in E \backslash E^{\prime}$ and $\operatorname{dep}(p), \operatorname{dep}(q)>s-m_{R}$, then there exists a path $q \stackrel{a^{-1}}{\longrightarrow}$ in $\mathcal{A}_{\varphi}^{\prime}$.

Proof. Clearly, we have a path $q \stackrel{a^{-1}}{\longrightarrow} p$ in $\mathcal{A}_{\varphi}$. We must show that all the edges in it are in $E^{\prime}$. First, we note that $a q$ must be reducible by definition of $E^{\prime}$, and so $q=a^{-1} u$ for some $u \in \overline{A^{*}} \backslash a A^{*}$.

Assume first that $a \in A_{1} \cup A_{1}^{-1}$. Suppose that $\left(q, a^{-1}, p\right) \notin E^{\prime}$. Then $a^{-1} p$ is reducible and so $p=a b v$ for some $b \in A \backslash\left\{a^{-1}\right\}$ and $v \in \overline{A^{*}}$. We have $|p|>$ $1+\left(m_{R}-1\right) d_{\varphi}$ by (3.2), hence $a^{-1} u=q=\overline{a^{-1} p(a \varphi)}=b \overline{v(a \varphi)}$, yielding $b=a^{-1}$ and contradicting $p=a b v$. Thus $\left(q, a^{-1}, p\right) \in E^{\prime}$ in this case.

Assume now that $a \in A_{0}$. For $i=1, \ldots, m_{a}-1$, we have an edge

$$
\overline{a^{m_{a}-i} u\left(a^{i-1} \varphi\right)} \stackrel{a}{\longrightarrow} \overline{a^{m_{a}-i-1} u\left(a^{i} \varphi\right)}
$$

in $\mathcal{A}_{\varphi}$. Since $|q| \geq s_{0}>1+\left(m_{R}-1\right) d_{\varphi}$ by $(3.2)$, we get $|u|>s_{0}-m_{a} \geq$ $\left(m_{R}-1\right)\left|a^{i-1} \varphi\right|,\left(m_{R}-1\right)\left|a^{i} \varphi\right|$. It follows that $a^{m_{a}-i} \overline{u\left(a^{i-1} \varphi\right)} \stackrel{a}{\longrightarrow} a^{m_{a}-i-1} \overline{u\left(a^{i} \varphi\right)}$ is an edge of $\mathcal{A}_{\varphi}$, indeed of $\mathcal{A}_{\varphi}^{\prime}$, for $i=1, \ldots, m_{a}-1$. Thus there is a path $q \stackrel{a^{-1}}{\longrightarrow} \overline{u\left(a^{m_{a}-1} \varphi\right)}$ in $\mathcal{A}_{\varphi}^{\prime}$. Since $\mathcal{A}_{\varphi}$ is deterministic, it follows that $\overline{u\left(a^{m_{a}-1} \varphi\right)}=p$ and the lemma is proved. 
Back to the proof of Theorem 3.2, let $\mathcal{B}_{\varphi}$ be the (finite) full subautomaton of $\mathcal{A}_{\varphi}$ induced by the subset of vertices of depth $\leq s$ (that is, $\mathcal{B}_{\varphi}$ contains all the edges of $\mathcal{A}_{\varphi}$ connecting vertices of depth $\left.\leq s\right)$.

Given $q \in Q$ of depth $>s-m_{R}$, by (3.1) and (3.2) there exists in $\mathcal{A}_{\varphi}^{\prime}$ a unique maximal path $\alpha_{q}: q \longrightarrow \ldots$ where every vertex has depth $>s-m_{R}$. Let $Q_{0}$ (respectively $Q_{1}$ ) denote the set of all $q \in Q$ with $s-m_{R}<\operatorname{dep}(q) \leq s$ such that the set of vertices occurring in $\alpha_{q}$ is finite (respectively infinite). Given $p, q \in Q_{1}$ distinct, let $p \wedge q$ denote the first vertex in $\alpha_{p}$ to appear in $\alpha_{q}$ (if such a vertex exists, otherwise $p \wedge q$ remains undefined). Then $p \wedge q=q \wedge p$, otherwise we would have a cycle

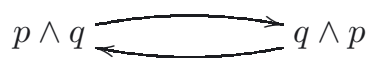

contradicting $p \in Q_{1}$.

We define $\mathcal{C}_{\varphi}$ to be the automaton obtained by adding to $\mathcal{B}_{\varphi}$ all vertices and edges in the following paths of $\mathcal{A}_{\varphi}^{\prime}$ :

(C1) $\alpha_{q}$ for $q \in Q_{0}$;

(C2) initial segments $q \longrightarrow p$ of $\alpha_{q}$ for $q \in Q_{1}$ and $\operatorname{dep}(p) \leq s$;

(C3) $p \longrightarrow p \wedge q$ for all $p, q \in Q_{1}$ such that $p \wedge q$ is defined.

Clearly, $\mathcal{C}_{\varphi}$ is finite. Finally, $\mathcal{C}_{\varphi}^{\prime}$ is obtained by adding to $\mathcal{C}_{\varphi}$, for every edge $(p, a, q)$ of $\mathcal{C}_{\varphi}$, all the edges in the path $q \stackrel{a^{-1}}{\longrightarrow} p$ in $\mathcal{A}_{\varphi}$. Note that $\mathcal{C}_{\varphi}^{\prime}$ is a finite subautomaton of $\mathcal{A}_{\varphi}$. Moreover, if $(p, a, q)$ is an edge of $\mathcal{C}_{\varphi}^{\prime}$, there exists a path $q \stackrel{a^{-1}}{\longrightarrow} p$ in $\mathcal{C}_{\varphi}^{\prime}$.

We prove now that

$$
\overline{\operatorname{Fix} \varphi} \subseteq L\left(\mathcal{C}_{\varphi}^{\prime}\right)
$$

Recall that $(\operatorname{Fix} \varphi) \pi^{-1}=L\left(\mathcal{A}_{\varphi}\right)$. Since $\mathcal{B}_{\varphi}$ is a subautomaton of $\mathcal{C}_{\varphi}^{\prime}$, it suffices to show that every path $p \stackrel{u}{\longrightarrow} q$ in $\mathcal{A}_{\varphi}$ such that $u \in \overline{A^{*}}, \operatorname{dep}(p)=s, \operatorname{dep}(q) \leq s$ and all intermediate vertices have depth $>s$, is also a path in $\mathcal{C}_{\varphi}^{\prime}$.

Let $p \stackrel{u}{\longrightarrow} q$ be such a path. We can factor this path as

$$
p=p_{0} \stackrel{u_{0}}{\longrightarrow} r_{1} \stackrel{v_{1}}{\longrightarrow} p_{1} \stackrel{u_{1}}{\longrightarrow} \ldots \stackrel{v_{n}}{\longrightarrow} p_{n} \stackrel{u_{n}}{\longrightarrow} r_{n+1}=q,
$$

where the $r_{i} \stackrel{v_{i}}{\longrightarrow} p_{i}$ group the edges in $E \backslash E^{\prime}$, and $u_{i}, v_{j} \neq 1$ for $1 \leq i \leq n-1$ and $1 \leq j \leq n$. Note that, when $(r, a, t) \in E$, then $\operatorname{dep}(t)>\operatorname{dep}(r)-m_{R}$ : this is clear if $a \in A_{1} \cup_{-1} A_{1}^{-1}$ since $\left(t, a^{-1}, r\right) \in E$; on the other hand, if $a \in A_{0}$, then there is a path $t \stackrel{a^{-1}}{\longrightarrow}$ of length $<m_{R}$ and our claim holds too. It follows that $\operatorname{dep}(q)>s-m_{R}$ and so we can apply Lemma 3.3 and get paths

$$
p=p_{0} \stackrel{u_{0}}{\longrightarrow} r_{1} \stackrel{v_{1}^{-1}}{\longleftarrow} p_{1} \stackrel{u_{1}}{\longrightarrow} \ldots \stackrel{v_{n}^{-1}}{\longleftarrow} p_{n} \stackrel{u_{n}}{\longrightarrow} r_{n+1}=q
$$

in $\mathcal{A}_{\varphi}^{\prime}$. Assume first that $n=0$. Then $p \stackrel{u}{\longrightarrow} q$ is an initial segment of some path $\alpha_{q}$ in $(\mathrm{C} 1)$ or $(\mathrm{C} 2)$, hence is a path in $\mathcal{C}_{\varphi}$ and therefore in $\mathcal{C}_{\varphi}^{\prime}$. Thus we assume that $n>0$. Take $i \in\{1, \ldots, n\}$. We show that $u_{i}$ is a proper prefix of $v_{i}^{-1}$.

Indeed, we have paths

$$
r_{i} \stackrel{v_{i}^{-1}}{\stackrel{u_{i}}{\longrightarrow}} p_{i+1}
$$


in $\mathcal{A}_{\varphi}^{\prime}$ and all our vertices are too deep to have outdegree $>1$. Hence one of this two paths must be an initial segment of the other. Write $v_{i}=x a$ with $a \in A$. If $a \in A_{1} \cup A_{1}^{-1}$, then $u_{i}=1$, otherwise $u_{i} \in a^{-1} A^{*}$, and $a a^{-1}$ would be a factor of $v_{i} u_{i}$, contradicting the irreducibility of $u$. Hence we may assume that $a \in A_{0}$ and so $v_{i}^{-1}=a^{m_{a}-1} x^{-1}$. Similarly to the preceding case, $u_{i} \in a^{m_{a}-1} A^{*}$ would contradict the irreducibility of $u$, hence $u_{i}$ must be a proper prefix of $a^{m_{a}-1}$ and therefore of $v_{i}^{-1}$.

Write $v_{i}^{-1}=u_{i} w_{i}$. We have paths

$$
p \stackrel{u_{0}}{\longrightarrow} r_{1} \stackrel{w_{1}}{\longleftarrow} r_{2} \stackrel{w_{2}}{\longleftarrow} \ldots \stackrel{w_{n}}{\stackrel{4}{\leftrightarrows}} r_{n+1}=q
$$

in $\mathcal{A}_{\varphi}^{\prime}$. Let $w=w_{n} w_{n-1} \ldots w_{1}$. We claim that $p \stackrel{u_{0}}{\longrightarrow} r_{1} \stackrel{w}{\longleftarrow} q$ are paths in $\mathcal{C}_{\varphi}^{\prime}$.

This is immediate if $p \in Q_{0}$ (which is equivalent to $q \in Q_{0}$ ), hence we assume that $p, q \in Q_{1}$.

Suppose that $p=q$. Then $u_{0}=w$ and so $w v_{1} u_{1}$ is a factor of $u$, hence irreducible. Decompose in letters $v_{1}=a_{1} \ldots a_{k}$. Since $u_{1} w_{1}=v_{1}^{-1}=a_{k}^{-1} \ldots a_{1}^{-1}$ and $w_{1} v_{1} u_{1}$ is irreducible, it follows that $k=1$, otherwise $a_{k}^{-1}$ is a prefix of $u_{1}$ or $a_{1}^{-1}$ is a suffix of $w_{1}$. If $a_{1} \notin A_{0}$, then $u_{1} w_{1}=a_{1}^{-1}$ produces immediately a contradiction. On the other hand, if $a_{1} \in A_{0}$, then $u_{1} w_{1}=a^{m_{a}-1}$ and so $w_{1} v_{1} u_{1}=a^{m_{a}}$ is reducible, another contradiction. Hence $p \neq q$.

Write $u_{0}=u_{0}^{\prime} h, w=w^{\prime} h$, where $h$ denotes the longest common suffix of the two words. Then we have paths $p \stackrel{u_{0}^{\prime}}{\longrightarrow} p \wedge q \stackrel{w^{\prime}}{\stackrel{\text { in }}{\longrightarrow}} \mathcal{C}_{\varphi}$. So we are done if $h=1$. Assume then that $h \neq 1$ and write $h=h^{\prime} b$ with $b \in A$. Note that, since $u_{1}$ is a proper prefix of $v_{1}^{-1}$, then $w_{1}$ is a nontrivial suffix of $w$. If $b \notin A_{0}$, then $w_{1} \in A^{*} b$ and so $v_{1} \in b^{-1} A^{*}$. Hence $b b^{-1}$ would be a factor of $u_{0} v_{1}$, contradicting the irreducibility of $u$. Hence $b \in A_{0}$. Write $h=h^{\prime \prime} b^{i}$ with $h^{\prime \prime} \notin A^{*} b$. Then $w_{1} \in A^{*} b$ and so $v_{1} \in b A^{*}$. Suppose first that $v_{1}=b v^{\prime} c$ with $c \in A$. Then $u_{1} w_{1}=v_{1}^{-1}=c^{-1} v^{\prime-1} b^{m_{b}-1}$. If $c^{-1}$ is a prefix of $u_{1}$, then $v_{1} u_{1}$ would not be irreducible, hence $b^{m_{b}-1}$ is a suffix of $w_{1}$. Now, since $u_{0} v_{1}$ is irreducible and $v_{1}$ starts by $b$, we have $i \leq m_{a}-2$ and so $h=b^{i}$. Moreover, since $b^{m_{b}-1}$ is a suffix of $w$, the last edge of $q \stackrel{w^{\prime}}{\longrightarrow} p \wedge q$ belongs to the $b$-labelled cycle of $\mathcal{A}_{\varphi}$ containing $p \wedge q \stackrel{h}{\longrightarrow} r_{1}$, and so the edges in this path must belong to $\mathcal{C}_{\varphi}^{\prime}$.

Assume now that $v_{1}=b$. Then $u_{1} w_{1}=b^{m_{b}-1}$. Since $u_{0} v_{1} u_{1}=u_{0}^{\prime} h^{\prime \prime} b^{i} b u_{1}$ is irreducible, we have $\left|w_{1}\right|>i$ and so once again the last edge of $q \stackrel{w^{\prime}}{\longrightarrow} p \wedge q$ belongs to the $b$-labelled cycle of $\mathcal{A}_{\varphi}$ containing $p \wedge q \stackrel{h}{\longrightarrow} r_{1}$. Therefore the edges in this path must belong to $\mathcal{C}_{\varphi}^{\prime}$.

Now, since $p \stackrel{u_{0}}{\longrightarrow} r_{1} \stackrel{w}{\longleftarrow} q$ are paths in $\mathcal{C}_{\varphi}^{\prime}$, also $r_{1} \stackrel{w^{-1}}{\longrightarrow} q$ is a path in $\mathcal{C}_{\varphi}^{\prime}$. Hence $v_{i}^{-1}=u_{i} w_{i}$ yields $v_{i}=\overline{w_{i}^{-1} u_{i}^{-1}}$ and so $\overline{w_{i}^{-1}}=\overline{v_{i} u_{i}}=v_{i} u_{i}$ since $v_{i} u_{i}$ is irreducible. Since $w^{-1}=w_{1}^{-1} \ldots w_{n}^{-1}$, it follows that there exists a path in $\mathcal{C}_{\varphi}^{\prime}$ of the form $r_{1} \stackrel{z}{\longrightarrow} q$ for $z=v_{1} u_{1} \ldots v_{n} u_{n}$. Therefore $p \stackrel{u}{\longrightarrow} q$ is a path in $\mathcal{C}_{\varphi}^{\prime}$ and $(3.3)$ holds.

Now $\overline{\operatorname{Fix} \varphi} \subseteq L\left(\mathcal{C}_{\varphi}^{\prime}\right) \subseteq L\left(\mathcal{A}_{\varphi}\right)=($ Fix $\varphi) \pi^{-1}$ yields $\overline{\operatorname{Fix} \varphi} \subseteq \overline{L\left(\mathcal{C}_{\varphi}^{\prime}\right)} \subseteq \overline{\text { Fix } \varphi}$ and so $\overline{\operatorname{Fix} \varphi}=\overline{L\left(\mathcal{C}_{\varphi}^{\prime}\right)}$. By Theorem 2.2, $\overline{\operatorname{Fix} \varphi}$ is rational and so $\operatorname{Fix} \varphi$ is a rational subset of $G$. By Proposition 2.1, Fix $\varphi$ is then a finitely generated subgroup of $G$. 
Corollary 3.4. Let $\varphi$ be an endomorphism of a finitely generated free product of a free monoid and cyclic groups. Then $\operatorname{Fix} \varphi$ is rational.

Proof. Let $M=A_{0}^{*} * G_{1}$, where $G_{1}$ is the c-free group defined by the finite special confluent rewriting system $\left\langle A_{1} \mid R_{1}\right\rangle$. Let $G$ be the c-free group defined by the finite special confluent rewriting system $\langle A \mid R\rangle$, where $A=A_{0} \cup A_{0}^{-1} \cup A_{1}$ and $R=R_{1} \cup\left\{\left(a a^{-1}, 1\right),\left(a^{-1} a, 1\right) \mid a \in A_{0}\right\}$. Let $\bar{u}$ (respectively $\widehat{u}$ ) denote the irreducible form of $u$ in the rewriting system of $M$ (respectively $G$ ).

Now $\varphi$ extends to an endomorphism $\Phi$ of $G$ through $a_{0}^{-1} \Phi=\left(a_{0} \varphi\right)^{-1}\left(a_{0} \in A_{0}\right)$. By Theorem 3.2, Fix $\Phi$ is a finitely generated and therefore rational subgroup of $G$. Denoting the canonical homomorphism $A^{*} \rightarrow G$ by $\pi$, it follows that $\operatorname{Fix} \Phi=L \pi$ for some rational $L \subseteq A^{*}$. In view of Theorem $2.2, \widehat{\operatorname{Fix} \Phi}=\widehat{L}$ is a rational subset of $A^{*}$. Since $\overline{\operatorname{Fix} \varphi}=\widehat{\operatorname{Fix} \Phi} \cap\left(A_{0} \cup A_{1}\right)^{*}$, it follows that $\overline{\operatorname{Fix} \varphi}$ is a rational subset of $\left(A_{0} \cup A_{1}\right)^{*}$ and so Fix $\varphi$ is a rational subset of $M$.

\section{Computability}

We start this section by considering the simple case of free monoid endomorphisms, whose discussion is essential to the follow-up. The first proof was given by Head [14], and an alternative one was later provided by Hamm and Shallit [13]. We include here a very short proof.

Let $A_{0}$ be a finite alphabet and let $\varphi \in \operatorname{End} A_{0}^{*}$. Write $m=\left|A_{0}\right|$ and define

$$
\begin{aligned}
& A_{2}=\left\{a \in A_{0} \mid a \varphi^{n}=1 \text { for some } n \geq 1\right\}, \\
& A_{3}=A_{0} \backslash A_{2}, \\
& A_{4}=\left\{a \in A_{3} \mid a \varphi \in A_{2}^{*} a A_{2}^{*}\right\} .
\end{aligned}
$$

Let $\Gamma$ be the directed graph with vertex set $A_{0}$ and edges $a \longrightarrow b$ whenever $b$ occurs in $a \varphi$. Then $a \in A_{2}$ if and only if there exists no infinite path $a \longrightarrow \cdots$ in $\Gamma$. This is equivalent to say there is no path $a \longrightarrow \cdots$ in $\Gamma$ of length $m$, hence

$$
A_{2}=\left\{a \in A_{0} \mid a \varphi^{m}=1\right\}
$$

and is therefore effectively computable, and so are $A_{3}$ and $A_{4}$.

Given $B \subseteq A$, we denote by $\theta_{A, B}$ the homomorphism $A^{*} \rightarrow B^{*}$ defined by

$$
a \theta= \begin{cases}a & \text { if } a \in B \\ 1 & \text { otherwise }\end{cases}
$$

Lemma 4.1 ([14]). Let $\varphi \in \operatorname{End} A_{0}^{*}$ and $m=\left|A_{0}\right|$. Then $\operatorname{Fix} \varphi=\left(A_{4} \varphi^{m}\right)^{*}$.

Proof. Let $a \in A_{4}$. Then $a \varphi=u a v$ for some $u, v \in A_{2}^{*}$. It follows from (4.1) that

$$
a \varphi^{m+1}=(u a v) \varphi^{m}=\overline{\left(u \varphi^{m}\right)\left(a \varphi^{m}\right)\left(v \varphi^{m}\right)}=a \varphi^{m},
$$

hence $A_{4} \varphi^{m} \subseteq \operatorname{Fix} \varphi$ and so $\left(A_{4} \varphi^{m}\right)^{*} \subseteq \operatorname{Fix} \varphi$.

To prove the opposite inclusion, let $\theta=\theta_{A_{0}, A_{3}}$. Take $u \in \operatorname{Fix} \varphi$. Then $u \varphi \theta=u \theta$. Since $A_{2} \varphi \subseteq A_{2}^{*}$, we get $u \theta \varphi \theta=u \varphi \theta=u \theta$ and so $u \theta \in \operatorname{Fix}(\varphi \theta)$. Since $1 \notin A_{3} \varphi \theta$, it follows easily that $u \theta \in A_{4}^{*}$. Hence $u \in\left(A_{2} \cup A_{4}\right)^{*}$ and so $u=u \varphi^{m} \in\left(A_{2} \cup\right.$ $\left.A_{4}\right)^{*} \varphi^{m}=A_{4}^{*} \varphi^{m}=\left(A_{4} \varphi^{m}\right)^{*}$ as required. 
Note that, given an endomorphism $\varphi$ of $A_{0}^{*} * G$, where $G$ is a group, the restriction $\left.\varphi\right|_{G}$ is an endomorphism of $G$. Clearly, $G$ is the (unique) maximal subgroup of $A_{0}^{*} * G$. To proceed with our task, we must be able to articulate the processes taking pace at the group components and the free monoid components. That is achieved through the next result, in circumstances which are far from general:

Theorem 4.2. Let $M=A_{0}^{*} * G$ be finitely generated, where $G$ is a c-free group. Let $\varphi \in \operatorname{End} M$ be such that the equation

$$
x=v\left(\left.x \varphi\right|_{G}\right) w \quad(x \in G)
$$

has an effectively constructible rational solution set for all $v, w \in G$. Then $\operatorname{Fix} \varphi$ is an effectively constructible rational submonoid of $M$.

Proof. Let $G$ be defined by the finite special confluent rewriting system $\left\langle A_{1} \mid R\right\rangle$. Write $A=A_{0} \cup A_{1}$ and $\theta=\theta_{A, A_{0}}$. For every $u \in A^{*} \backslash A_{1}^{*}$, let $u \xi$ denote the longest factor of $u$ in $A_{0} A^{*} \cap A^{*} A_{0}$. Let $A_{2}, A_{3}$ and $A_{4}$ be defined as in the beginning of the section, replacing $A$ by $A_{0}$ and $\varphi$ by $\psi=\left.\varphi\right|_{A_{0}^{*}} \theta$. Write $m=\left|A_{0}\right|$. Let $u=a_{1} \ldots a_{n} \in A_{4} \psi^{m}=\operatorname{Fix} \psi$ with $a_{1}, \ldots, a_{n} \in A_{0}$.

Our proof of Theorem 4.2 requires the following intermediate result:

Lemma 4.3. There exist effectively constructible rational subsets $L_{1}, \ldots, L_{n-1}$ of $G$ such that the solution set of the equation

$$
a_{1} x_{1} a_{2} \ldots x_{n-1} a_{n}=\left(a_{1} x_{1} a_{2} \ldots x_{n-1} a_{n}\right) \varphi \xi \quad\left(x_{i} \in G\right)
$$

is precisely $L_{1} \times \ldots \times L_{n-1}$.

Proof. Let $1 \leq i_{1}<\ldots<i_{k} \leq n$ denote all $i \in\{1, \ldots, n\}$ such that $a_{i} \psi \neq 1$. Then there exist $1=j_{1}<\ldots<j_{k+1}=n+1$ such that

$$
a_{i_{r}} \psi=a_{j_{r}} \ldots a_{j_{r+1}-1}
$$

for every $r \in\{1, \ldots, k\}$. Moreover, there exist words $p_{s}, w_{s}, q_{s} \in \overline{A_{1}^{*}}$ (for all adequate values of $s$ ) such that

$$
a_{i_{r}} \varphi=p_{j_{r}-1} a_{j_{r}} w_{j_{r}} a_{j_{r}+1} \ldots w_{j_{r+1}-2} a_{j_{r+1}-1} q_{j_{r+1}-1}
$$

for $r \in\{1, \ldots, k\}$. We claim that equation (4.2) is equivalent to the system of $n-1$ equations

$$
\left\{\begin{array}{l}
x_{j_{r}-1}=q_{j_{r}-1}\left(x_{i_{r-1}} a_{i_{r-1}+1} x_{i_{r-1}+1} \ldots a_{i_{r}-1} x_{i_{r}-1}\right) \varphi p_{j_{r}-1} \\
\quad \text { for } r \in\{2, \ldots, k\} \\
x_{i}=w_{i} \quad \text { whenever } j_{r} \leq i<j_{r+1}-1 \text { for some } r \in\{1, \ldots, k\} .
\end{array}\right.
$$

Indeed, since $a_{1} \ldots a_{n} \in$ Fix $\psi$, all we need is to find out necessary and sufficient conditions that the $x_{i}$ must satisfy in equation (4.2). Let $i \in\{1, \ldots, n-1\}$, and 
consider $x_{i}$ in the left hand side of (4.2). Clearly, if $j_{r} \leq i<j_{r+1}-1$ for some $r \in\{1, \ldots, k\}$, then $x_{i}$ is fully determined by $a_{j_{r}} \varphi$ through $x_{i}=w_{i}$.

Otherwise, $i=j_{r}-1$ for some $r \in\{2, \ldots, k\}$. In this case, we need to compute which part of the right hand side of (4.2) eventually determines $x_{j_{r}-1}$ : the longest suffix of $a_{i_{r-1}} \varphi$ in $\overline{A_{1}^{*}}$, the longest prefix of $a_{i_{r}} \varphi$ in $\overline{A_{1}^{*}}$, and the image of the factor between $a_{i_{r-1}}$ and $a_{i_{r}}$. This proves the claim.

Next we show that our system is equivalent to a system of equations of the form $\left(x_{i}=y_{i}\right)_{i=1, \ldots, n-1}$, where

$$
y_{i} \in \overline{A_{1}^{*}} \cup \overline{A_{1}^{*}}\left(x_{i} \varphi\right) \overline{A_{1}^{*}}
$$

for $i=1, \ldots, n-1$. For that purpose, we define a directed graph $\Lambda$ with vertex set $\{1, \ldots, n-1\}$ and edges

$$
\left(j_{r}-1\right) \longrightarrow i \quad \text { whenever } i_{r-1} \leq i<i_{r}(r=2, \ldots, k) .
$$

Clearly, if $j$ has outdegree 0 , then $x_{j}=w_{j}$ is an equation in the original system. It follows easily that, if there is no infinite path $j \longrightarrow \cdots$ in $\Lambda$, then $x_{j}$ is uniquely determined in the solution set of (4.2) (if it is nonempty). Thus we need to discuss the structure of infinite paths in $\Lambda$. Since $\Lambda$ is finite, such an infinite path must always contain a cycle. We show next that all cycles in $\Lambda$ have length 1 .

Suppose that

$$
\left(j_{r_{1}}-1\right) \longrightarrow\left(j_{r_{2}}-1\right) \longrightarrow \cdots \longrightarrow\left(j_{r_{s}}-1\right)=\left(j_{r_{1}}-1\right)
$$

is a cycle of length $s-1$ in $\Lambda$. We claim that there exists a loop $\left(j_{r_{1}}-1\right) \longrightarrow\left(j_{r_{1}}-1\right)$ in $\Lambda$, which is equivalent to

$$
i_{r_{1}-1} \leq j_{r_{1}}-1<i_{r_{1}}
$$

Suppose first that $i_{r_{1}-1}>j_{r_{1}}-1$. It suffices to show that

$$
i_{r_{t}-1}>j_{r_{t}}-1 \text { implies }\left(r_{t}<r_{t+1} \text { and } i_{r_{t+1}-1}>j_{r_{t+1}}-1\right) \text { for } t=1, \ldots, s-1
$$

to derive a contradiction from $r_{s}=r_{1}$. Indeed, assume that $i_{r_{t}-1}>j_{r_{t}}-1$. Since $\left(j_{r_{t}}-1\right) \longrightarrow\left(j_{r_{t+1}}-1\right)$ is an edge of $\Lambda$, we have $i_{r_{t}-1} \leq j_{r_{t+1}}-1<i_{r_{t}}$ and so $i_{r_{t}-1}<j_{r_{t+1}} \leq i_{r_{t}}$. Hence $j_{r_{t}} \leq i_{r_{t}-1}<j_{r_{t+1}}$ and so $r_{t}<r_{t+1}$. It follows that $i_{r_{t+1}-1} \geq i_{r_{t}}>j_{r_{t+1}}-1$ and so our implication holds, yielding the desired contradiction.

Suppose now that $j_{r_{1}}-1 \geq i_{r_{1}}$. Similarly to the preceding case, it suffices to show that

$$
j_{r_{t}}-1 \geq i_{r_{t}} \text { implies }\left(r_{t}>r_{t+1} \text { and } j_{r_{t+1}}-1 \geq i_{r_{t+1}}\right) \text { for } t=1, \ldots, s-1
$$

to derive a contradiction from $r_{s}=r_{1}$. Indeed, assume that $j_{r_{t}}-1 \geq i_{r_{t}}$. Since $i_{r_{t}-1} \leq j_{r_{t+1}}-1<i_{r_{t}}$, we get $j_{r_{t}}-1 \geq i_{r_{t}}>j_{r_{t+1}}-1$ and so $r_{t}>r_{t+1}$. It follows that $j_{r_{t+1}}-1 \geq i_{r_{t}-1} \geq i_{r_{t+1}}$, yielding the desired contradiction. 
Thus (4.3) holds and so there exists a loop $\left(j_{r_{1}}-1\right) \longrightarrow\left(j_{r_{1}}-1\right)$ in $\Gamma$. It is immediate that no vertex of $\Lambda$ can have indegree $>1$, hence $j_{r_{s-1}}-1=j_{r_{1}}-1$ and so $r_{s-1}=r_{1}$, yielding $s=2$. Therefore all cycles in $\Lambda$ have length 1 .

In view of the indegree property, it follows that the unique infinite path $j \longrightarrow \cdots$ in $\Lambda$, if such a path exists, consists of infinitely many tours of the loop $j \longrightarrow j$ : you cannot enter a loop unless you have always been there.

Now take an equation of the form

$$
x_{j_{r}-1}=q_{j_{r}-1}\left(x_{i_{r-1}} a_{i_{r-1}+1} x_{i_{r-1}+1} \ldots a_{i_{r}-1} x_{i_{r}-1}\right) \varphi p_{j_{r}-1}
$$

for $r \in\{2, \ldots, k\}$ in our original system. If there is no loop $j_{r}-1 \longrightarrow j_{r}-1$ in $\Lambda$, then the equations $x_{i}=w_{i}$ will eventually determine a unique possible value for $x_{j_{r}-1}$ and we can replace the above equation by some other of the form $x_{j_{r}-1}=w_{j_{r}-1}$ for some $w_{j_{r}-1} \in \overline{A_{1}^{*}}$.

Finally, assume that there is a loop $j_{r}-1 \longrightarrow j_{r}-1$ in $\Lambda$. Then $x_{j_{r}-1} \in$ $\left\{x_{i_{r-1}}, \ldots, x_{i_{r}-1}\right\}$ and all the other variables are bound to be eventually determined by the equations $x_{i}=w_{i}$. Since

$$
q_{j_{r}-1}, a_{i_{r-1}+1} \varphi, \ldots, a_{i_{r}-1} \varphi, p_{j_{r}-1} \in \overline{A_{1}^{*}}
$$

are constants, we can replace our equation by another of the form $x_{j_{r}-1}=$ $v_{j_{r}-1}\left(x_{j_{r}-1} \varphi\right) z_{j_{r}-1}$ for some $v_{j_{r}-1}, z_{j_{r}-1} \in \overline{A_{1}^{*}}$. Note also that this construction of the new system is an effective procedure since it consists of successively replacing some $x_{i}$ by $w_{i}$ in the other equations. Therefore we can now concentrate on the new system $\left(x_{i}=y_{i}\right)_{i=1, \ldots, n-1}$. Since each variable occurs just in one equation, and no equation contains more than one single variable, the solution set will come out as a direct product $L_{1} \times \ldots \times L_{n-1}$, where $L_{i}$ is the solution set of the equation containing $x_{i}$. If this equation is of the form $x_{i}=w_{i}$, then $L_{i}=\left\{w_{i}\right\}$ is (trivially) an effectively constructible rational subset of $G$. If the equation is of the form $x_{i}=v_{i}\left(x_{i} \varphi\right) z_{i}$, then the claim follows from the Lemma's hypothesis on $\left.\varphi\right|_{G}$.

Back to the proof of Theorem 4.2, we remark that there exist $p, q \in G$ such that

$$
\left(a_{1} x_{1} a_{2} \ldots x_{n-1} a_{n}\right) \varphi=p\left(\left(a_{1} x_{1} a_{2} \ldots x_{n-1} a_{n}\right) \varphi \xi\right) q
$$

for every solution $\left(x_{1}, \ldots, x_{n-1}\right) \in L_{1} \times \ldots \times L_{n-1}$. Indeed, the prefix erased by $\xi$ is $\left(a_{1} x_{1} \ldots a_{i_{1}-1} x_{i_{1}-1}\right) \varphi v_{i_{1}}$ and is uniquely determined since $x_{1}, x_{2}, \ldots, x_{i_{1}-1}$ are uniquely determined as well. A symmetric argument applies to suffixes.

Back to the proof of Theorem 4.2, take $u \in \overline{A^{*}}$. Note that $u \in$ Fix $\varphi$ implies $u \theta \in \operatorname{Fix} \psi$, hence we may restrict our attention to this latter condition. In view of Lemma 4.1, we may write $u \theta=u_{1} \ldots u_{t}$ for some $u_{1}, \ldots, u_{t} \in A_{4} \psi^{m}$. If $t=0$, then $\left.\varphi\right|_{G} \in \operatorname{End} G$ implies that $u \in \operatorname{Fix} \varphi$ if and only if $\left.u \in \operatorname{Fix} \varphi\right|_{G}$, and we can use the theorem's hypothesis with $v=w=1$. Therefore we only need to concentrate on the case $t>0$.

Write

$$
u_{i}=a_{i 1} a_{i 2} \ldots a_{i n_{i}} \quad\left(a_{i j} \in A_{0}\right)
$$


for $i=1, \ldots, t$. Then we may consider

$$
u=y_{0} a_{11} x_{11} a_{12} x_{12} \ldots a_{1 n_{1}} y_{1} a_{21} x_{21} \ldots a_{2 n_{2}} y_{2} \ldots y_{t-1} a_{t 1} x_{t 1} \ldots a_{t n_{t}} y_{t}
$$

with $y_{i}, x_{i j} \in G$.

Now $u_{1}, \ldots, u_{t} \in \operatorname{Fix} \psi$. Moreover, for every $i \in\{1, \ldots, t\}$, by the remark following the proof of Lemma 4.3, there exist $p_{i}, q_{i} \in G$ such that

$$
\left(a_{i 1} x_{i 1} a_{i 2} x_{i 2} \ldots a_{i n_{i}}\right) \varphi=p_{i}\left(\left(a_{i 1} x_{i 1} a_{i 2} x_{i 2} \ldots a_{i n_{i}}\right) \varphi \xi\right) q_{i}
$$

for every solution of the equation $a_{i 1} x_{i 1} a_{i 2} x_{i 2} \ldots a_{i n_{i}}=\left(a_{i 1} x_{i 1} a_{i 2} x_{i 2} \ldots a_{i n_{i}}\right) \varphi \xi$. We call $p_{i}$ (respectively $q_{i}$ ) the volatile prefix (respectively suffix) of the equation associated to $a_{i 1} \ldots a_{i n_{i}}$.

Using the conventions $q_{0}=p_{t+1}=1$, it is straightforward to check that $u \in$ Fix $\varphi$ if and only

$$
\left\{\begin{array}{c}
a_{i 1} x_{i 1} a_{i 2} x_{i 2} \ldots a_{i n_{i}}=\left(a_{i 1} x_{i 1} a_{i 2} x_{i 2} \ldots a_{i n_{i}}\right) \varphi \xi \\
\text { for } i=1, \ldots, t \\
y_{i}=q_{i}\left(y_{i} \varphi_{G}\right) p_{i+1} \\
\text { for } i=0, \ldots, t
\end{array}\right.
$$

Note that, in such a system, the solution sets of the blocks of equations $a_{i 1} x_{i 1} a_{i 2} x_{i 2} \ldots a_{i n_{i}}=\left(a_{i 1} x_{i 1} a_{i 2} x_{i 2} \ldots a_{i n_{i}}\right) \varphi \xi(i=1, \ldots, t)$ have effectively constructible rational solution sets in view of Lemma 4.3. On the other hand, each equation $y_{i}=q_{i}\left(y_{i} \varphi_{G}\right) p_{i+1}$ has also an effectively constructible rational solution set by the theorem's hypothesis, and there are only finitely many choices for the $p_{i}, q_{i}$.

Assume that $A_{4} \psi^{m}=\left\{f_{1}, \ldots, f_{s}\right\}$. For $i=1, \ldots, s$, let $L_{1} \times \cdots \times L_{n-1}$ denote the solution set of equation (4.2), where $f_{i} \theta=a_{1} a_{2} \ldots a_{n}$. We define $Y_{i}=a_{1} L_{1} a_{2} \ldots L_{n-1} a_{n}$. Note that $Y_{i}$ is an effectively constructible rational subset of $M$.

For $i, j=1, \ldots, s$, we define the following subsets of $G$, where $p_{i}$ (respectively $q_{i}$ ) is the volatile prefix (respectively suffix) of the equation associated to $f_{i}$ :

- $Z_{i j}$ is the solution set of the equation $y=q_{i}\left(y \varphi_{G}\right) p_{j}$;

- $Z_{i, s+1}$ is the solution set of the equation $y=q_{i}\left(y \varphi_{G}\right)$;

- $Z_{0 j}$ is the solution set of the equation $y=\left(y \varphi_{G}\right) p_{j}$;

- $Z_{0, s+1}=\operatorname{Fix} \varphi_{G}$.

By the theorem's hypothesis, all the $Z_{i j}$ are effectively constructible rational subsets of $G$.

We build an $A$-automaton with rational edges $\mathcal{A}=\left(Q, f_{0}, f_{s+1}, E\right)$ as follows:

- $Q=\left\{f_{0}, f_{1}, \ldots, f_{s}, f_{s+1}\right\}$

- for $i=1, \ldots, s$ and $j=1, \ldots, s+1,\left(f_{i}, Y_{i} Z_{i j}, f_{j}\right) \in E$;

- for $j=1, \ldots, s+1,\left(f_{0}, Z_{0 j}, f_{j}\right) \in E$.

It is straightforward to check that $\operatorname{Fix} \varphi=(L(\mathcal{A})) \pi$, hence Fix $\varphi$ is an effectively constructible rational submonoid of $M$. 
We can now obtain the corollary to follow. We would prefer of course to pass of the restriction on $\left.\varphi\right|_{G}$, but that reflects the present state of things at the free group level.

Corollary 4.4. Let $M=A_{0}^{*} * G$ be finitely generated, where $G$ is a free group. Let $\varphi \in \operatorname{End} M$ be such that $\left.\varphi\right|_{G}$ is an automorphism. Then $\operatorname{Fix} \varphi$ is an effectively constructible rational submonoid of $M$.

Proof. In view of Theorem 4.2, it suffices to prove that the equation

$$
x=v(x \psi) w \quad(x \in G)
$$

has an effectively constructible rational solution set $L$ for all $v, w \in G$ and $\psi \in$ Aut $G$.

Fix $v, w, \psi$. Then $x=v(x \psi) w$ is equivalent to $x^{-1} v(x \psi)=w^{-1}$ and so we can decide whether or not this equation has a solution $x_{0}$ by [4], and compute it in the affirmative case. Now

$$
\begin{aligned}
x=v(x \psi) w & \Leftrightarrow x^{-1} v(x \psi)=w^{-1} \Leftrightarrow x^{-1} v(x \psi)=x_{0}^{-1} v\left(x_{0} \psi\right) \\
& \Leftrightarrow x_{0} x^{-1}=v\left(x_{0} x^{-1}\right) \psi v^{-1} \\
& \Leftrightarrow x_{0} x^{-1}=\left(x_{0} x^{-1}\right) \psi \lambda_{v^{-1}} \Leftrightarrow x x_{0}^{-1}=\left(x x_{0}^{-1}\right) \psi \lambda_{v^{-1}}
\end{aligned}
$$

where $\lambda_{v^{-1}}$ denotes the inner automorphism of $G$ defined by $g \lambda_{v^{-1}}=v g v^{-1}$. Since $\psi \lambda_{v^{-1}} \in \operatorname{Aut} G$, it follows from [16] that Fix $\left(\psi \lambda_{v^{-1}}\right)$ is an effectively constructible finitely generated subgroup of $G$, and so $L=\left(\operatorname{Fix}\left(\psi \lambda_{v^{-1}}\right)\right) x_{0}$ is an effectively constructible rational subset of $G$ as required.

The next example shows that $\operatorname{Fix} \varphi$ is not necessarily a finitely generated submonoid of $M$. Note that Fix $\varphi$ is finitely generated if $M$ is a free monoid (by Lem. 4.1) or a free group (by [10]).

Example 4.5. Let $M=\{a, c\}^{*} \times F G_{\{b\}}$ and let $\varphi \in$ End $M$ be defined by $a \varphi=a b$, $b \varphi=b$ and $c \varphi=b^{-1} c$. Then $\left.\varphi\right|_{F G_{\{b\}}}$ is an automorphism but Fix $\varphi$ is not finitely generated.

Let $G=F G_{\{b\}}$. It is a simple exercise to show that Fix $\varphi=(G \cup a G c)^{*}$ and that this monoid is not finitely generated.

Acknowledgements. The author acknowledges support from the European Regional Development Fund through the programme COMPETE and by the Portuguese Government through FCT (Fundação para a Ciência e a Tecnologia) under the project PEst-C/MAT/UI0144/2011, and also Project ASA (PTDC/MAT/65481/2006). 


\section{REFERENCES}

[1] M. Benois, Descendants of regular language in a class of rewriting systems: algorithm and complexity of an automata construction, in Proc. of RTA 8\%. Lect. Notes Comput. Sci. 256 (1987) 121-132.

[2] J. Berstel, Transductions and Context-free Languages. Teubner, Stuttgart (1979).

[3] M. Bestvina and M. Handel, Train tracks and automorphisms of free groups. Ann. Math. 135 (1992) 1-51.

[4] O. Bogopolski, A. Martino, O. Maslakova and E. Ventura, The conjugacy problem is solvable in free-by-cyclic groups. Bull. Lond. Math. Soc. 38 (2006) 787-794.

[5] R.V. Book and F. Otto, String-Rewriting Systems. Springer-Verlag, New York (1993).

[6] J. Cassaigne and P.V. Silva, Infinite words and confluent rewriting systems: endomorphism extensions. Int. J. Algebra Comput. 19 (2009) 443-490.

[7] J. Cassaigne and P.V. Silva, Infinite periodic points of endomorphisms over special confluent rewriting systems. Ann. Inst. Fourier 59 (2009) 769-810.

[8] D.J. Collins and E.C. Turner, Efficient representatives for automorphisms of free products. Mich. Math. J. 41 (1994) 443-464.

[9] D. Cooper, Automorphisms of free groups have finitely generated fixed point sets. J. Algebra 111 (1987) 453-456.

[10] S.M. Gersten, Fixed points of automorphisms of free groups. Adv. Math. 64 (1987) 51-85.

[11] R.Z. Goldstein and E.C. Turner, Monomorphisms of finitely generated free groups have finitely generated equalizers. Invent. Math. 82 (1985) 283-289.

[12] R.Z. Goldstein and E.C. Turner, Fixed subgroups of homomorphisms of free groups. Bull. Lond. Math. Soc. 18 (1986) 468-470.

[13] D. Hamm and J. Shallit, Characterization of finite and one-sided infinite fixed points of morphisms on free monoids. Technical Report CS-99-17 (1999).

[14] T. Head, Fixed languages and the adult languages of 0L schemes. Int. J. Comput. Math. 10 (1981) 103-107.

[15] S. Lyapin, Semigroups. Fizmatgiz. Moscow (1960). English translation by Am. Math. Soc. (1974).

[16] O.S. Maslakova, The fixed point group of a free group automorphism. Algebra i Logika 42 (2003) 422-472. English translation in Algebra Logic 42 (2003) 237-265.

[17] M. Petrich and P.V. Silva, On directly infinite rings. Acta Math. Hung. 85 (1999) 153-165.

[18] J. Sakarovitch, Éléments de Théorie des Automates. Vuibert, Paris (2003).

[19] P.V. Silva, Rational subsets of partially reversible monoids. Theoret. Comput. Sci. 409 (2008) 537-548.

[20] P.V. Silva, Fixed points of endomorphisms over special confluent rewriting systems. Monatsh. Math. 161 (2010) 417-447.

[21] M. Sykiotis, Fixed subgroups of endomorphisms of free products. J. Algebra 315 (2007) $274-278$.

[22] E. Ventura, Fixed subgroups of free groups: a survey. Contemp. Math. 296 (2002) 231-255.

Communicated by G. Richomme.

Received November 2, 2010. Accepted October 3, 2011. 